\title{
Competitive Online Algorithms for Resource Allocation over the Positive Semidefinite Cone
}

\author{
Reza Eghbali* $\quad$ James Saunderson ${ }^{\dagger} \quad$ Maryam Fazel $^{\ddagger}$
}

April 22, 2022

\begin{abstract}
We consider a new and general online resource allocation problem, where the goal is to maximize a function of a positive semidefinite (PSD) matrix with a scalar budget constraint. The problem data arrives online, and the algorithm needs to make an irrevocable decision at each step. Of particular interest are classic experiment design problems in the online setting, with the algorithm deciding whether to allocate budget to each experiment as new experiments become available sequentially.

We analyze two greedy primal-dual algorithms and provide bounds on their competitive ratios. Our analysis relies on a smooth surrogate of the objective function that needs to satisfy a new diminishing returns (PSD-DR) property (that its gradient is order-reversing with respect to the PSD cone). Using the representation for monotone maps on the PSD cone given by Löwner's theorem, we obtain a convex parametrization of the family of functions satisfying PSD-DR. We then formulate a convex optimization problem to directly optimize our competitive ratio bound over this set. This design problem can be solved offline before the data start arriving. The online algorithm that uses the designed smoothing is tailored to the given cost function, and enjoys a competitive ratio at least as good as our optimized bound. We provide examples of computing the smooth surrogate for D-optimal and A-optimal experiment design, and demonstrate the performance of the custom-designed algorithm.
\end{abstract}

\section{Introduction}

Online resource allocation problems and algorithms have been traditionally studied in operations research (e.g., revenue management [AWY14] and references therein), online network routing [BN06] and computer science (e.g., online packing and covering in linear or convex case [BN09b, $\left.\mathrm{ABC}^{+} 16\right]$, online welfare maximization [KPV13]). In recent years, applications in online advertising such as the Adwords problem [MSVV07] have garnered renewed interest in this topic. In all these applications, the demands for resources arrive in an online, sequential fashion, and resource allocation also happens online. For example, in online revenue management, customers arrive sequentially, reveal their demand for the resources and offer a bid price. The inventory owner then needs to make an irrevocable decision about the latest customer's offer, without knowing future demands, while aiming to maximize his or her revenue. A feature in these problems is that the variables denoting allocation amounts, the bid prices, and the coefficients in the resource constraints are all

\footnotetext{
${ }^{*}$ Tetration Analytics, Cisco Systems, Inc. Palo Alto, CA 94301 reghbali@cisco.com, eghbali@uw.edu

${ }^{\dagger}$ Department of Electrical and Computer Systems Engineering, Monash University, Victoria 3800, Australia, james.saunderson@monash.edu

${ }^{\ddagger}$ Department of Electrical Engineering, University of Washington, Seattle, WA 98195 mfazel@uw. edu
} 
nonnegative, thus a resource can only be used up as time goes on (resource usage is nondecreasing) while the total available resource is fixed. In the context of linear programming (LP), these are sometimes called "packing" problems, e.g., [MR13, BN09b]. For an overview of online linear programming and more generally online convex problems with different models for online information arrival, we refer the reader to [LJ16, GM16, AD15, ESF14].

In this paper, we consider a new and general online resource allocation problem, where the objective is a function of a positive semidefinite (PSD) matrix (thus the problem is defined on the PSD cone $S_{+}^{n}$ ), with a scalar budget constraint. The problem can be described as follows: At round $t$, the algorithm receives a matrix $A_{t} \in S_{+}^{n}$ and scalar $c_{t}>0$, and then chooses $x_{t} \in[0,1]$. The goal of the algorithm is to maximize $H\left(\sum_{t=1}^{m} A_{t} x_{t}\right)$ subject to the budget constraint $\sum_{t=1}^{m} c_{t} x_{t} \leq b$. The offline problem can be written as

$$
\text { maximize } H\left(\sum_{t=1}^{m} A_{t} x_{t}\right) \quad \text { subject to }\left\{\begin{array}{l}
\sum_{t=1}^{m} c_{t} x_{t} \leq b, \\
0 \leq x_{t} \leq 1, t=1, \ldots, m .
\end{array}\right.
$$

We assume that $H: S_{+}^{n} \rightarrow \mathbf{R}$ is a monotone, concave trace function, i.e.,

$$
H(X)=\sum_{i=1}^{n} h\left(\lambda_{i}(X)\right)
$$

where $h: \mathbf{R}_{+} \rightarrow \mathbf{R}$ is a monotone concave function and $\lambda_{i}(X)$ denotes the $i$ th eigenvalue of $X$. Note that analogous to packing LPs, $\sum_{s=1}^{t} A_{s} x_{s}$ is nondecreasing with respect to the PSD cone. In other words,

$$
\sum_{s=1}^{t} A_{s} x_{s} \preceq \sum_{s=1}^{t+1} A_{s} x_{s} \quad \text { for all } t
$$

where $X \preceq Y$ means that $Y-X$ is positive semidefinite.

In the online algorithms literature, various scenarios have been considered for the information being revealed to the algorithm (the sequence of $A_{t}$ and $c_{t}$ ): from the worst-case adversarial model to various stochastic models. In this paper, since we are interested in understanding the limits of performance and providing competitive ratio bounds, we assume an adversarial model, where little is known about the arriving data. One could use techniques similar to ours in other scenarios as well, which we leave for future work.

Our setup covers online versions of problems such as sensor selection or experiment design, and graph formation [MFM16]. In these problems, $A_{t}=a_{t} a_{t}^{T}$ for some $a_{t} \in \mathbf{R}^{n}$. In online experiment design, the vector $a_{t}$ is an experiment or measurement vector that provides linear noisy measurements of an unknown vector $w$, i.e., $\xi_{t}=\left\langle a_{t}, w\right\rangle+n_{t}$, where $n_{t}$ is Gaussian noise and $c_{t} x_{t}$ is the experiment cost. In this paper, we consider two algorithms: one that assigns fractional values to $x_{t}$ and one that assign integer values to $x_{t}$. The algorithm makes online decisions about the budget to allocate to an experiment, aiming to minimize various functions of the error covariance matrix of the maximum a priori estimate of $w$.

The online experiment design problem can be expressed as (1), with different choices of $H$ giving rise to different criteria for optimal experiment design. Examples include $H(X)=\log \operatorname{det}(\epsilon I+X)$ for the D-optimal criterion, $H(X)=-\operatorname{tr}(\epsilon I+X)^{-1}$ for the A-optimal criterion, and $H(X)=$ $-\operatorname{tr}(\epsilon I+X)^{-p}$ for the $p$ th mean criterion. (The prior distribution on $w$ is assumed to be $\mathcal{N}\left(0, \frac{1}{\epsilon} I\right)$.)

We use the A-optimal and D-optimal criteria, as well as the problem where $H(X)=\operatorname{tr}(X)$, as running examples throughout the paper. The latter simply recovers a linear program with one packing constraint, and provides insight into the connections and differences with online Linear Programming results, e.g., [BN09b] (discussed in section 3). 
Our results exploit a crucial property of the objective function $H$ or its surrogate $H_{S}$ (discussed in section 3.2) which we refer to as the PSD diminishing returns property. For a function with a scalar variable, this property simply means the larger the variable, the smaller the derivative. More generally, we define it as follows.

Definition 1 (PSD Diminishing Returns). A concave trace function $F$ satisfies PSD diminishing returns (PSD-DR) if $\nabla F$ is order-reversing with respect to $S_{+}^{n}$, i.e.,

$$
\nabla F(U) \preceq \nabla F\left(U^{\prime}\right), \text { whenever } U \succeq U^{\prime} \text {. }
$$

Our contributions. Our focus is on developing algorithms with (multiplicative) competitive ratio guarantees for problems of the form (1). In other words, our algorithms provide feasible solutions $x_{1}, x_{2}, \ldots, x_{m}$ such that $H\left(\sum_{t=1}^{m} A_{t} x_{t}\right)-H(0) \geq \operatorname{cr}\left(P^{\star}-H(0)\right)$, where cr bounds the competitive ratio and $P^{\star}$ is the optimal value of $(1)^{1}$.

The algorithms we consider (Algorithms 1 and 2, in Section 2) are parameterized by a smoothed surrogate, $H_{S}$, of the objective function $H$, and a smoothed penalty function $G_{S}$ in place of the budget constraint $\sum_{t=1}^{m} x_{t} \leq b$. Our main contribution is a systematic method, based on solving an offline convex optimization problem, to design $H_{S}$ and $G_{S}$, and to compute a bound on the competitive ratio of the associated algorithms.

Our approach builds on the results in [EF16a], which considers a general online conic optimization problem and shows that smoothing the objective function (to obtain a surrogate) before applying a primal-dual greedy algorithm improves the competitive ratio. However, prior work was not able to address the problem of finding the best surrogate (or even representing the space of suitable surrogates) - except for the special (and restrictive) case of separable objectives defined on the non-negative orthant, which reduces to a search over concave scalar functions. There is no hope of employing a similar strategy for functions defined on the PSD cone, unless one has access to representations that lead to a convex parametrization for PSD-DR functions. The present paper resolves this issue.

We require the smoothed objective $H_{S}$ to satisfy the PSD-DR property. Note that this does not hold for all concave trace functions (indeed fails to hold for the objective function in A-optimal experiment design). If the objective function in problem (1) does not satisfy this property, our approach allows us to design an appropriate surrogate $H_{S}$ that satisfies it, and use this $H_{S}$ to construct algorithms with competitive ratio guarantees. And if the original $H$ does satisfy the property, our approach still helps improve the competitive ratio; see section 3.2. A key observation in this paper is that we can use Löwner's theorem, characterizing operator monotone functions, to impose this constraint in a computationally effective way by requiring it to have a certain integral representation (see (17)).

The rest of the paper is structured as follows. In Section 2, we describe the algorithms. In Sections 3 and 4, we provide the competitive ratio analysis for the online algorithms described in Section 2 and determine how the competitive ratio depends on the smoothed surrogate of the objective function. We set up optimization problems for finding surrogates that maximize the competitive ratio bound derived in these sections. Numerical examples are presented in Section 5. Related works are discussed in section 6.

\footnotetext{
${ }^{1}$ To simplify the notation in the rest of the paper, assume $H(0)=0$ by replacing $h(u)$ with $h(u)-h(0)$
} 


\section{Algorithms}

In this section we describe the class of online algorithms we consider to solve (1), and give bounds on the duality gap achieved by these algorithms. The algorithms we consider (Algorithms 1 and 2, stated in Section 2.1) are (modified) greedy algorithms, where a primal-dual greedy approach is applied to a smoothed surrogate for the objective. These algorithms are instances of the general algorithmic framework for conic online optimization introduced in [EF16a], which can recover stateof-the-art algorithms developed for Adwords [BJN07], Adwords with concave returns [DJ12], and online LP [BN09a].

Before describing the algorithms, it is helpful to slightly rewrite (1) and state its dual. Throughout, let $G: \mathbf{R}_{+} \rightarrow \mathbf{R}$ be the indicator function of the interval $[0, b]$, i.e.,

$$
G(u)= \begin{cases}0 & \text { if } u \in[0, b] \\ -\infty & \text { otherwise }\end{cases}
$$

Then we can rewrite (1) as

$$
\underset{x_{1}, \ldots, x_{m} \in[0,1]}{\operatorname{maximize}} H\left(\sum_{t=1}^{m} A_{t} x_{t}\right)+G\left(\sum_{t=1}^{m} c_{t} x_{t}\right) .
$$

To derive a dual program, we rewrite the primal as:

$$
\begin{aligned}
\underset{x_{1}, \ldots, x_{m} \in[0,1]}{\operatorname{maximize}} H(U)+G(u) . \\
\text { subject to } U=\sum_{t=1}^{m} A_{t} x_{t} \\
u=\sum_{t=1}^{m} c_{t} x_{t}
\end{aligned}
$$

Introducing a dual matrix variable $Y$ and dual scalar variable $z$ corresponding to the two equality constraints, we can write the Lagrangian as follows:

$$
\mathcal{L}\left(u, U, x_{1}, \ldots, x_{m}, Y, z\right)=H(U)-\langle Y, U\rangle+G(u)-\langle z, u\rangle+\sum_{t=1}^{m}\left(\left\langle A_{t}, Y\right\rangle+c_{t} z\right) x_{t}
$$

where $\operatorname{dom}(\mathcal{L})=\mathbf{S}^{n} \times \mathbf{R} \times[0,1]^{m} \times \mathbf{S}^{n} \times \mathbf{R}$. Maximizing $\mathcal{L}$ over all the primal variables, we can derive the dual of (1) in terms of conjugate functions as

$$
\underset{z, Y}{\operatorname{minimize}} \sum_{t=1}^{m}\left(\left\langle A_{t}, Y\right\rangle+c_{t} z\right)_{+}-H^{*}(Y)-G^{*}(z),
$$

where, for a function $\psi: \mathbf{R}^{n} \rightarrow \mathbf{R}, \psi^{*}$ denotes the concave conjugate of $\psi$, defined as $\psi^{*}(y)=$ $\inf _{u}\langle y, u\rangle-\psi(u)$. The concave conjugate of $G$ is

$$
G^{*}(z)= \begin{cases}b z & \text { if } z \leq 0 \\ 0 & \text { otherwise }\end{cases}
$$

For a trace function $H(U)=\sum_{i=1}^{n} h\left(\lambda_{i}(U)\right)$ we have that $H^{*}(Y)=\sum_{i=1}^{n} h^{*}\left(\lambda_{i}(Y)\right)$, a result established in [Lew95]. 


\subsection{Statement of algorithms}

Recall that our objective function $H$ is a monotone, concave, trace function, and that $G$ is the concave indicator function of the set $[0, b]$. We consider two online algorithms for (1). Algorithm 1 makes sequential updates to the primal and dual variables, whereas Algorithm 2 makes these updates simultaneously. These algorithms require a choice of functions $H_{S}$ and $G_{S}$ that are smooth surrogates for $H$ and $G$. Throughout, we assume these functions satisfy the following.

Assumption 1 (Assumptions on $H_{S}$ ). We assume that $h_{S}: O \rightarrow \mathbf{R}$ is concave, differentiable, and satisfies $h_{S}(0)=0$ and $h_{S}^{\prime}(0)=h^{\prime}(0)$, where $O$ is an open interval that contains $\mathbf{R}_{+}$. We assume that $H_{S}: S_{+}^{n} \rightarrow \mathbf{R}$ is the trace function $H_{S}(U)=\sum_{i=1}^{n} h_{S}\left(\lambda_{i}(U)\right)$.

Assumption 2 (Assumptions on $G_{S}$ ). We assume that $G_{S}: O \rightarrow \mathbf{R}$ is concave, differentiable, and satisfies $G_{S}(0)=0$ and $G_{S}^{\prime}(0)=0$, where $O$ is an open interval that contains $\mathbf{R}_{+}$.

The problem of designing $H_{S}$ and $G_{S}$, given $H$ and $G$, is the main focus of the paper, and is the subject of Section 3. For now we merely point out that the results of this section hold for any choice of $H_{S}$ and $G_{S}$ satisfying the basic assumptions above.

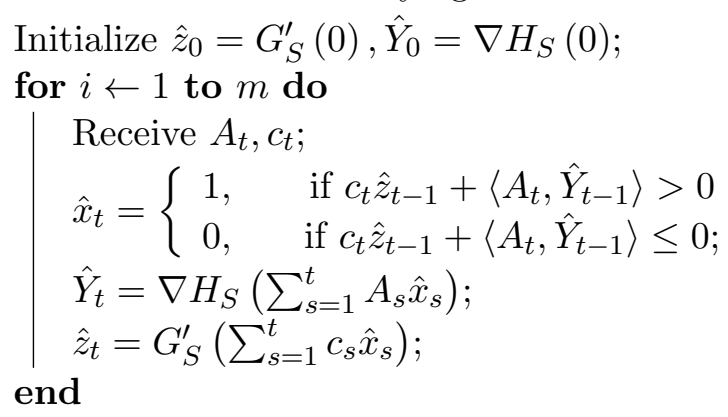

Algorithm 1: Sequential Update

for $i \leftarrow 1$ to $m$ do

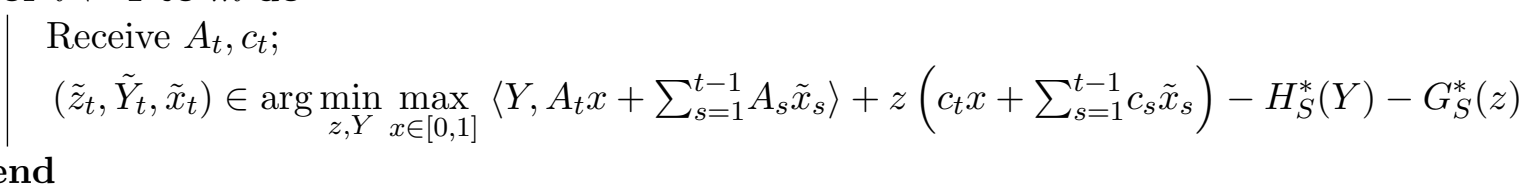

Algorithm 2: Simultaneous Update

In Algorithm 2, at each step the primal variable $\tilde{x}_{t}$, and the dual variables $\tilde{Y}_{t}$ and $\tilde{z}_{t}$, are updated together, by finding the saddle point of the Lagrangian (solution to the min-max problem). To argue for the existence of a saddle point, we use Corollary 11.41b in [RWW98] which requires two conditions to be satisfied. The first condition is that the following problem has a nonempty, bounded set of maximizers,

$$
\tilde{x}_{t} \in \arg \max _{x \in[0,1]} H_{S}\left(A_{t} x+\sum_{s=1}^{t-1} A_{s} \tilde{x}_{s}\right)+G_{S}\left(x+\sum_{s=1}^{t-1} \tilde{x}_{s}\right),
$$

which holds because $[0,1]$ is a compact convex set. The second condition requires

$$
\begin{aligned}
& \sum_{s=1}^{t-1} A_{s} \tilde{x}_{s} \in \operatorname{int}\left(\left\{-A_{t} x \mid x \in[0,1]\right\}+\operatorname{dom} H_{S}\right), \\
& \sum_{s=1}^{t-1} c_{s} \tilde{x}_{s} \in \operatorname{int}\left(\left\{-c_{t} x \mid x \in[0,1]\right\}+\operatorname{dom} G_{S}\right),
\end{aligned}
$$


where the sum is the Minkowski sum of two sets, and int denotes the interior of a set. This condition is also satisfied since 0 is in the interior of the domains of $h_{S}$ and $G_{S}$ (from Assumptions (1) and $(2))$.

Algorithm 1 can also be interpreted in similar terms, by observing that the primal $\hat{x}_{t}$ update can be written as

$$
\hat{x}_{t} \in \underset{x \in[0,1]}{\operatorname{argmax}} x\left(c_{t} \hat{z}_{t-1}+\left\langle A_{t}, \hat{Y}_{t-1}\right\rangle\right),
$$

and using the fact that for a differentiable concave function $\psi$, we have $\nabla \psi(u)=\arg \min _{y}\langle y, u\rangle-$ $\psi^{*}(u)$, the dual updates can be written as

$$
\begin{aligned}
& \hat{z}_{t}=\underset{z}{\operatorname{argmin}} z\left(\sum_{s=1}^{t} c_{s} \hat{x}_{s}\right)-G_{S}^{*}(z) \quad \text { and } \\
& \hat{Y}_{t}=\underset{Y}{\operatorname{argmin}}\left\langle Y, \sum_{s=1}^{t} A_{s} \hat{x}_{s}\right\rangle-H_{S}^{*}(Y),
\end{aligned}
$$

As such, the sequential algorithm can be viewed as alternating over maximization and minimization of the Lagrangian over primal and dual variables.

Algorithm 1, assigns integer values to $\hat{x}_{t}$ and the dual variable $\hat{z}_{t}$ acts as a decision threshold in the assignment rule for $\hat{x}_{t}$. For the reader familiar with online learning, we point out a connection with the typical online learning setup. The $\hat{Y}_{t}$ update step is the same as Follow-the-RegularizedLeader (FTRL) update with $H_{S}$ as the regularizer, so this algorithm is running FTRL on the dual problem.

Algorithm 2 clearly requires more computation than the sequential algorithm, but it is also easier to bound its competitive ratio. We do not focus on implementation details of this algorithm in this paper, but we point out that one can decide how accurately to solve the saddle-point subproblem at each iteration, trading off computation with desired accuracy (for each subproblem). To analyze this more practical variation of Algorithm 2, our analysis should take into account how solving each saddle-point problem to $\epsilon$ accuracy affects the overall competitive ratio. We leave this additional analysis to future work.

\subsection{Duality gap}

The competitive analysis, and subsequent design of $H_{S}$ and $G_{S}$ (in Section 3), relies on bounding the duality gap. Define the dual value achieved by the sequential and simultaneous algorithms by

$$
\begin{aligned}
& D_{\text {seq }}=\sum_{t=1}^{m}\left(\left\langle A_{t}, \hat{Y}_{t-1}\right\rangle+c_{t} \hat{z}_{t-1}\right)_{+}-H^{*}\left(\hat{Y}_{m}\right)-G^{*}\left(\hat{z}_{m}\right) \\
& D_{\text {sim }}=\sum_{t=1}^{m}\left(\left\langle A_{t}, \tilde{Y}_{t}\right\rangle+c_{t} \tilde{z}_{t}\right)_{+}-H^{*}\left(\tilde{Y}_{m}\right)-G^{*}\left(\tilde{z}_{m}\right) .
\end{aligned}
$$

The following two lemmas are essentially taken from [EF16b]. We include proofs in Appendix A to make the present paper self-contained. Lemma 1 bounds the duality gap, and Lemma 2 relates $D_{\text {seq }}$ and $D_{\text {sim }}$ to the dual optimal value $D^{\star}$, given an additional assumption on the gradient of $H_{S}$.

Lemma 1. Let $\tilde{x}_{t}, \tilde{Y}_{t}$, and $\tilde{z}_{t}$ and $\hat{x}_{t}, \hat{Y}_{t}$, and $\hat{z}_{t}$ denote the th iterate of the simultaneous and sequential algorithms, respectively. Then the duality gaps for the two algorithms satisfy the lower bounds

$$
\begin{aligned}
H_{S}\left(\sum_{t=1}^{m} A_{t} \tilde{x}_{t}\right)+G_{S}\left(\sum_{t=1}^{m} c_{t} \tilde{x}_{t}\right)-D_{\text {sim }} \geq & H^{*}\left(\tilde{Y}_{m}\right)+G^{*}\left(\tilde{z}_{m}\right) . \\
H_{S}\left(\sum_{t=1}^{m} A_{t} \hat{x}_{t}\right)+G_{S}\left(\sum_{t=1}^{m} c_{t} \hat{x}_{t}\right)-D_{\text {seq }} \geq & H^{*}\left(\hat{Y}_{m}\right)+G^{*}\left(\hat{z}_{m}\right)+ \\
& \sum_{t=1}^{m}\left\langle A_{t} \hat{x}_{t}, \hat{Y}_{t}-\hat{Y}_{t-1}\right\rangle+\sum_{t=1}^{m}\left\langle c_{t} \hat{x}_{t}, \hat{z}_{t}-\hat{z}_{t-1}\right\rangle .
\end{aligned}
$$


Proof. See Appendix A.

This duality gap bound is reminiscent of regret bounds in online learning. In regret analysis one directly bounds the right hand side of (6) in terms of the horizon and problem parameters. However, bounding the competitive ratio requires more assumptions and further analysis. The PSD Diminishing Returns (PSD-DR) property is used to relate $D_{\text {seq }}$ and $D_{\text {sim }}$ to the dual optimal value $D^{\star}$.

Lemma 2. If $H_{S}$ satisfies $P S D-D R$, then $D_{\mathrm{seq}} \geq D^{\star}$ and $D_{\mathrm{sim}} \geq D^{\star}$.

For completeness the proof is given in Appendix A. We remark that the PSD-DR property is a special case of the property used in the abstract framework of [EF16a] that was applied to general concave functions on proper cones. In order to develop computational methods to design the function $H_{S}$ (in Section 3.2), we will need to impose the PSD-DR property on $H_{S}$ in a computationally tractable way. A central observation of this paper is that this is possible for the PSD-DR property by exploiting a celebrated theorem of Löwner. We discuss this in detail in Section 3.2.

\section{Competitive ratio analysis}

In this section we show how to design $H_{S}$ and $G_{S}$ in the sequential algorithm (Algorithm 2) to maximize a lower bound on the competitive ratio achieved. We discuss the appropriate modifications for the sequential algorithm in Section 4. In what follows, $e=2.718 \ldots$ is Euler's number.

We first state a technical lemma (Lemma 3 ) that relates the budget consumed and competitive ratio achieved by Algorithm 2 to the functions $G_{S}$ and $H_{S}$. The only dependence on the data (the values of $A_{t}$ and $c_{t}$ ) is via two parameters, $\theta$ and $\Theta$, which are lower and upper bounds on $c_{t}^{-1} \operatorname{tr}\left(A_{t}\right)$ for all $t$. We use a parameter $\gamma \geq 1$ as a trade-off parameter between the budget consumption and competitive ratio. The result says that if $H_{S}, G_{S}$ satisfy certain inequalities that involve $\gamma$ and $\beta$, then Algorithm 2 achieves a competitive ratio of at least $\frac{1}{\gamma /(e-1)+\beta}$ using a budget of $G_{S}^{\prime-1}\left(-h^{\prime}(0) \Theta\right)$ (the inverse function of the derivative of $G_{S}$ evaluated at a point). Concrete values for these bounds are worked out at the end of this section. This is a similar style of result to [BN09a], which applies to a special class of linear programs.

Lemma 3. Let $G$ be as defined in (2), and let $h$ be concave and monotonically increasing, with corresponding trace function $H$. Let $G_{S}$ satisfy Assumption 2, $h_{S}$ satisfy Assumption 1, and suppose that the corresponding trace function $H_{S}$ satisfies the PSD-DR property. Suppose that $\theta \leq c_{t}^{-1} \operatorname{tr}\left(A_{t}\right) \leq \Theta$ for all $t$ and $u_{\max } \geq \lambda_{\max }\left(\sum_{t=1}^{m} A_{t} \tilde{x}_{t}\right){ }^{2}$ Then

1. If for a given $\gamma \geq 1, G_{S}$ satisfies

$$
\gamma G_{S}(u) \leq G^{*}\left(G_{S}^{\prime}(u)\right)+\frac{\gamma}{e-1} h(\theta u) \quad \text { for all } u \in[0, \infty),
$$

and there exists $\beta>0$ such that $h_{S}$ satisfies

$$
\gamma h_{S}(u) \leq h^{*}\left(h_{S}^{\prime}(u)\right)+\beta h(u) \quad \text { for all } u \in\left[0, u_{\max }\right]
$$

then the iterates $\tilde{x}_{1}, \ldots, \tilde{x}_{m}$ of Algorithm 2 satisfy

$$
H\left(\sum_{t=1}^{m} A_{t} \tilde{x}_{t}\right) \geq \frac{1}{\gamma /(e-1)+\beta} D^{\star} .
$$

\footnotetext{
${ }^{2}$ Note that we could choose, for instance, $u_{\max }=b^{\prime} \max _{t} c_{t}^{-1} \lambda_{\max }\left(A_{t}\right)$, but for certain classes of problems better bounds may be available.
} 
2. The iterates $\tilde{x}_{1}, \ldots, \tilde{x}_{m}$ of Algorithm 2 also satisfy

$$
\sum_{t=1}^{m} c_{t} \tilde{x}_{t} \leq b^{\prime}:=\inf \left\{u: G_{S}^{\prime}(u) \leq-h^{\prime}(0) \Theta\right\} .
$$

Before providing a proof of Lemma 3, we explain how we use it in what follows. As mentioned before, we consider $\gamma$ a design parameter that trades off between (possible) budget violation and competitive ratio achieved. The smaller the parameter $\gamma$, the larger the competitive ratio (evident from (8) and (9)) and the larger the budget violation. The role of $\gamma$ in the amount of budget consumed and the reason for requiring $\gamma \geq 1$ is discussed in Section 3.1. In Section 3.1 we use (7) and (10) in Lemma 3 to design $G_{S}$ to minimize the budget $b^{\prime}$ consumed for a given $\gamma$. This design problem requires the parameter $\theta$ only. The parameter $\gamma$ allows us to decouple the design of $G_{S}$ and $h_{S}$.

In Section 3.2, we use (8) and (9) in Lemma 3 to design $H_{S}$ via solving a convex optimization problem that maximizes the bound on competitive ratio (by minimizing $\beta$ ). This design problem requires the parameter $u_{\max }$ only.

Proof of Lemma 3. First we show that $\sum_{t=1}^{m} c_{t} \tilde{x}_{t} \leq b^{\prime}$. To do this we use the optimality conditions of the saddle point problem in Algorithm 2. Indeed, for any $t$, we have that

$$
\tilde{Y}_{t}=\nabla H_{S}\left(\sum_{s=1}^{t} A_{s} \tilde{x}_{s}\right) \quad \text { and } \quad \tilde{z}_{t}=G_{S}^{\prime}\left(\sum_{s=1}^{t} c_{s} \tilde{x}_{s}\right)
$$

and that $\left\langle\tilde{Y}_{t}, A_{t}\right\rangle+c_{t} \tilde{z}_{t}<0$ implies $\tilde{x}_{t}=0$.

Arguing by contradiction, let $T$ be the smallest index such that $\sum_{t=1}^{T} c_{t} \tilde{x}_{t}>b^{\prime}$. Then, by the definition of $b^{\prime}$ we have that $\tilde{z}_{T}=G_{S}^{\prime}\left(\sum_{t=1}^{T} c_{t} \tilde{x}_{t}\right)<-h^{\prime}(0) \Theta$. Observe that $\sum_{t=1}^{T} A_{t} \tilde{x}_{t} \succeq 0$, from which it follows, from the fact that $H_{S}$ is PSD-DR, that $\nabla H_{S}\left(\sum_{t=1}^{T} A_{t} \tilde{x}_{t}\right) \preceq \nabla H_{S}(0)=h_{S}^{\prime}(0) I$. Then

$$
\left\langle\tilde{Y}_{T}, A_{T}\right\rangle+c_{T} \tilde{z}_{T}=\left\langle\nabla H_{S}\left(\sum_{t=1}^{T} A_{t} \tilde{x}_{t}\right), A_{T}\right\rangle+c_{T} \tilde{z}_{T} \leq h_{S}^{\prime}(0) \operatorname{tr} A_{T}+c_{T} \tilde{z}_{T}<0
$$

where, for the last inequality, we use the fact that $h_{S}^{\prime}(0)=h^{\prime}(0)$ and that $c_{T}^{-1} \operatorname{tr} A_{T} \leq \Theta$. It follows from the optimality conditions that $\tilde{x}_{T}=0$. But then $\sum_{t=1}^{T-1} c_{t} \tilde{x}_{t}>b^{\prime}$, contradicting our choice of $T$. It follows that $\sum_{t=1}^{m} c_{t} \tilde{x}_{t} \leq b^{\prime}$.

We now turn our attention to the bound on the competitive ratio. Let $U=\sum_{t=1}^{m} A_{t} \tilde{x}_{t}$ and $u=\sum_{t=1}^{m} c_{t} \tilde{x}_{t}$, so that $\tilde{Y}_{m}=\nabla H_{S}(U)$, and $\tilde{z}_{m}=G_{S}^{\prime}(u)$. By the duality gap bound (Lemma 1) we have

$$
H_{S}(U)+G_{S}(u)-D_{\operatorname{sim}} \geq H^{*}\left(\tilde{Y}_{m}\right)+G^{*}\left(\tilde{z}_{m}\right)
$$

By the primal allocation rule in Algorithm 2, we have $\tilde{x}_{t}\left(c_{t} \tilde{z}_{t}+\left\langle A_{t}, \tilde{Y}_{t}\right\rangle\right) \geq 0$. Combining this observation with the concavity of $H_{S}$ and $G_{S}$, we get

$$
\begin{array}{r}
H_{S}\left(\sum_{s=1}^{t} A_{s} \tilde{x}_{s}\right)+G_{S}\left(\sum_{s=1}^{t} c_{s} \tilde{x}_{s}\right)-H_{S}\left(\sum_{s=1}^{t-1} A_{s} \tilde{x}_{s}\right)-G_{S}\left(\sum_{s=1}^{t-1} c_{s} \tilde{x}_{s}\right) \\
\geq \tilde{x}_{t}\left(c_{t} \tilde{z}_{t}+\left\langle A_{t}, \tilde{Y}_{t}\right\rangle\right) \geq 0
\end{array}
$$

By taking the sum over $t$ and telescoping the sum we get

$$
H_{S}\left(\sum_{s=1}^{m} A_{s} \tilde{x}_{s}\right)+G_{S}\left(\sum_{s=1}^{m} c_{s} \tilde{x}_{s}\right) \geq 0 .
$$


The last preparatory observation we need is the inequality $H(U) \geq h(\theta u)$. To see why this holds, note that because $h$ is monotonically increasing, and $\theta \leq c_{t}^{-1} \operatorname{tr}\left(A_{t}\right)$ for all $t$,

$$
\begin{aligned}
h(\theta u)=h\left(\theta \sum_{t=1}^{m} c_{t} \tilde{x}_{t}\right) & \leq h\left(\operatorname{tr}\left(\sum_{t=1}^{m} A_{t} \tilde{x}_{t}\right)\right) \\
& =h(\operatorname{tr}(U)) \leq \sum_{i=1}^{n} h\left(\lambda_{i}(U)\right)=H(U)
\end{aligned}
$$

where the last inequality holds because $h$ is concave and $h(0) \geq 0$ so $h$ is subadditive on $[0, \infty)$. Now we can write

$$
\begin{array}{ll}
H(U)-D_{\text {sim }} & \\
\geq-H_{S}(U)-G_{S}(u)+H^{*}(Y)+G^{*}(z)+H(U) & \text { By (11) } \\
\geq-H_{S}(U)+(\gamma-1) G_{S}(u)+\left(1-\frac{\gamma}{e-1}\right) H(U)+H^{*}(Y) & \text { By (7) and (13) } \\
\geq-H_{S}(U)+(1-\gamma) H_{S}(U)+\left(1-\frac{\gamma}{e-1}\right) H(U)+H^{*}(Y) & \text { By (12) and } \gamma \geq 1 \\
=-\gamma H_{S}(U)+\left(1-\frac{\gamma}{e-1}\right) H(U)+H^{*}(Y) & \\
\geq\left(1-\frac{\gamma}{e-1}-\beta\right) H(U) & \text { By (8). }
\end{array}
$$

Then the result follows from Lemma 2.

These results allow us to search for functions $h_{S}$ and $G_{S}$ that satisfy the assumptions of Lemma 3. We use $\gamma$ as a design parameter, controlling the trade-off between competitive ratio and the (possible) budget violation.

\subsection{Smoothing the budget penalty function $G$, taking $h$ into account.}

For a fixed choice of $\gamma$, our aim is to design $G_{S}$ so as to minimize $b^{\prime}$. We do so by explicitly constructing $G_{S}^{\prime}(u)$ such that $(7)$ is satisfied with equality for all $u \geq 0$, and showing that such a $G_{S}^{\prime}$ is optimal, in the sense that for a given $\gamma \geq 1$, defining $G_{S}$ via (14) minimizes the bound $b^{\prime}$ on the budget consumed by the simultaneous algorithm.

Proposition 1. Let $\bar{G}_{S}$ be any function that satisfies (7). Let ${ }^{3}$

$$
G_{S}^{\prime}(u)=-\frac{\gamma}{b(e-1)} \int_{0}^{u} \exp \left(\frac{\gamma}{b}(u-v)\right) \theta h^{\prime}(\theta v) d v \quad \text { for } u \geq 0
$$

Then $\bar{G}_{S}^{\prime}(u) \geq G_{S}^{\prime}(u)$ for all $u \geq 0$ and so

$$
\inf \left\{u: G_{S}^{\prime}(u) \leq-h^{\prime}(0) \Theta\right\} \leq \inf \left\{u: \bar{G}_{S}^{\prime}(u) \leq-h^{\prime}(0) \Theta\right\} .
$$

Proof. Clearly $G_{S}^{\prime}(u) \leq 0$ for all $u \geq 0$, and so for any $u \geq 0$, we have that $G^{*}\left(G_{S}^{\prime}(u)\right)=b G_{S}^{\prime}(u)$. We now explain why $G_{S}^{\prime}$ satisfies (7) with equality. (This can also be verified by direct substitution.) Because $G_{S}(0)=0$ (by assumption), it is enough to show that $G_{S}^{\prime}$ satisfies the linear, constant coefficient differential equation obtained by equating, and then differentiating, both sides of (7). After rearranging terms, this differential equation is

$$
G_{S}^{\prime \prime}(u)=\frac{\gamma}{b} G_{S}^{\prime}(u)-\frac{\gamma}{b(e-1)} \theta h^{\prime}(\theta u) .
$$

\footnotetext{
${ }^{3}$ We can extend the domain of $G_{S}$ to negative reals by letting $G_{S}=0$ on $\mathbf{R}_{-}$to satisfy the technical assumption on the domain of $G_{S}$ in Assumption (2).
} 
The function $G_{S}^{\prime}(u)$ that satisfies this equation for $u \in[0, \infty)$ together with $G^{\prime}(0)=0$, is given by the convolution of $-\frac{\gamma}{b(e-1)} \theta h^{\prime}(\theta u)$ with $e^{\gamma u / b}$, as in (14).

Since $G_{S}^{\prime}$ satisfies (7) with equality, it follows from Gronwall's inequality (see [Dra03] Corollary 2) that for any other function $\bar{G}_{S}$ that satisfies $(7)$, we have $\bar{G}_{S}^{\prime}(u) \geq G_{S}^{\prime}(u)$ for all $u \geq 0$.

Remark 1. Note that the function $G_{S}$ is a convolution of $h^{\prime}(\theta u)$ with an exponential function. It can also be viewed as a function derived by applying Nesterov's smoothing technique to G. To show this, we have to argue that $G_{S}^{*}-G^{*}$ is a concave function. Note that $G^{*}$ is linear over $\mathbf{R}_{-}$; therefore, $G_{S}^{*}-G^{*}$ is concave on the positive reals with $G_{S}^{*}(0)-G^{*}(0)=0$, and for $y>0, G_{S}^{*}(y)-G^{*}(y)=0$. This establishes the concavity of $G_{S}^{*}-G^{*}$. As we discuss in Example 1 below, $G_{S}^{*}-G^{*}$ has a closed form when $h$ is a linear function. However, for more general examples of $h$ we do not have a closed form for $G_{S}^{*}-G^{*}$.

Now, to justify the fact that we require $\gamma \geq 1$, we find a lower bound $G_{S}^{\prime}$ given in (14) in terms of $\gamma$ :

$$
\begin{aligned}
G_{S}^{\prime}(u) & =-\frac{\gamma}{b(e-1)} \exp \left(\frac{\gamma}{b} u\right) \int_{0}^{u} \exp \left(-\frac{\gamma}{b} v\right) \theta h^{\prime}(\theta v) d v \\
& \geq-\frac{\gamma \theta h^{\prime}(0)}{b(e-1)} \exp \left(\frac{\gamma}{b} u\right) \int_{0}^{u} \exp \left(-\frac{\gamma}{b} v\right) d v=-\frac{\theta h^{\prime}(0)\left(\exp \left(\frac{\gamma}{b} u\right)-1\right)}{e-1}
\end{aligned}
$$

The inequality above is exact if $h$ is linear, e.g., $H(U)=\operatorname{tr}(U)$. Now we can bound $b^{\prime}$ as:

$$
b^{\prime}=\inf \left\{u: G_{S}^{\prime}(u) \leq-h^{\prime}(0) \Theta\right\} \geq \frac{b}{\gamma} \log \left(\frac{\Theta}{\theta}(e-1)+1\right)
$$

This shows that if $\gamma<1$, then even in the trivial case where $\theta=\Theta$ and $h$ is linear the algorithm can go over budget.

We now compute $G_{S}^{\prime}$, and bound the budget $b^{\prime}$ consumed by the simultaneous algorithm using $G_{S}^{\prime}$, for three examples.

Example 1 (Linear objective function). Consider the linear function $h(u)=u$ which translates to $H(U)=\operatorname{tr}(U)$. This case allows us to show that our approach specializes to recover known results. In this case, the problem reduces to a linear program with one budget constraint,

$$
\text { maximize } \sum_{t=1}^{m} \operatorname{tr}\left(A_{t}\right) x_{t} \quad \text { subject to } \quad\left\{\begin{array}{l}
\sum_{t=1}^{m} c_{t} x_{t} \leq b \\
0 \leq x_{t} \leq 1
\end{array} \text { for } t=1,2, \ldots, m .\right.
$$

Computing the integral (14) gives $G_{S}^{\prime}(u)=\theta\left(1-\exp \left(\frac{\gamma}{b} u\right)\right) /(e-1)$. This choice of $G_{S}^{\prime}$ corresponds to the exponential update algorithm for online LP [BN09a], and, in this particular case, $G_{S}$ can also be derived as a smooth surrogate for $G$ using Nesterov smoothing with a shifted entropy as the proximity function [EF16a]. In this case the bound $b^{\prime}$ on the budget consumed is given by

$$
b^{\prime}=\inf \left\{u \mid G_{S}^{\prime}(u)<-h^{\prime}(0) \Theta\right\}=\frac{b}{\gamma} \log \left((e-1) \frac{\Theta}{\theta}+1\right) .
$$

Choosing $\gamma \geq \log \left((e-1) \frac{\Theta}{\theta}+1\right)$ ensures that the budget is not violated.

Example 2 (D-optimal experiment design). Suppose that $h(u)=\log (u+1)$, the objective function of interest in D-optimal experiment design. In this case $h^{\prime}(u)=(1+u)^{-1}$, and we note that it is possible to express the optimal $G_{S}^{\prime}$ from (14) in terms of special functions called exponential 
integrals. To obtain an upper bound on $b^{\prime}$, and hence on the budget consumed, we use the fact that $h^{\prime}(\theta v)=(1+\theta v)^{-1} \geq e^{-\theta v}$ whenever $\theta v \geq 0$. Using this inequality in (14), and computing the resulting (elementary) integral, we obtain the bound

$$
G_{S}^{\prime}(u) \leq \frac{1}{(e-1)} \frac{e^{-\theta u}-e^{\frac{\gamma}{b} u}}{\theta^{-1}+(b / \gamma)} \leq \frac{1}{(e-1)} \frac{1-e^{\frac{\gamma}{b} u}}{\theta^{-1}+(b / \gamma)} .
$$

Rearranging and using the definition of $b^{\prime}$ we see that

$$
b^{\prime} \leq \frac{b}{\gamma} \log \left[(e-1) \Theta\left(\theta^{-1}+b / \gamma\right)+1\right] .
$$

Since $\gamma \geq 1$, we can ensure that there is no budget violation, i.e., $b^{\prime} \leq b$, by choosing $\gamma \geq$ $\log \left[(e-1) \Theta\left(\theta^{-1}+b\right)+1\right]$. Figure 1a shows examples of $G_{S}$ for two values of $\gamma$ when $h(u)=$ $\log (1+u)$.

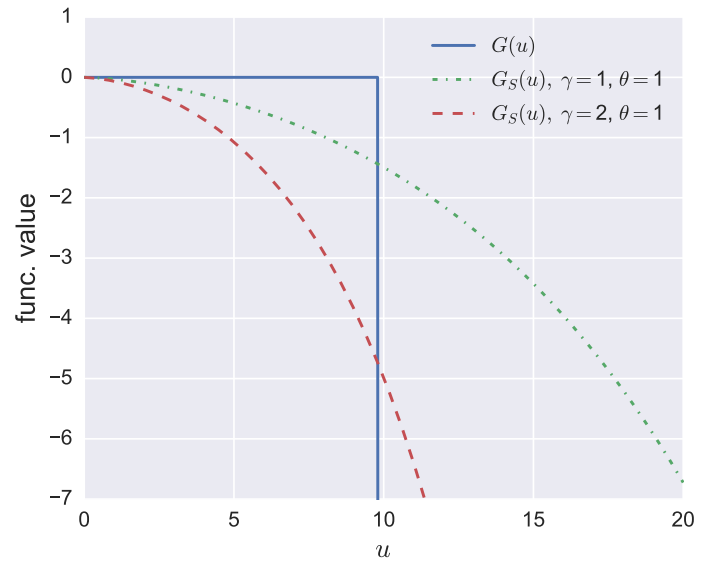

(a) Examples of $G_{S}$ when $h(u)=\log (u+1)$

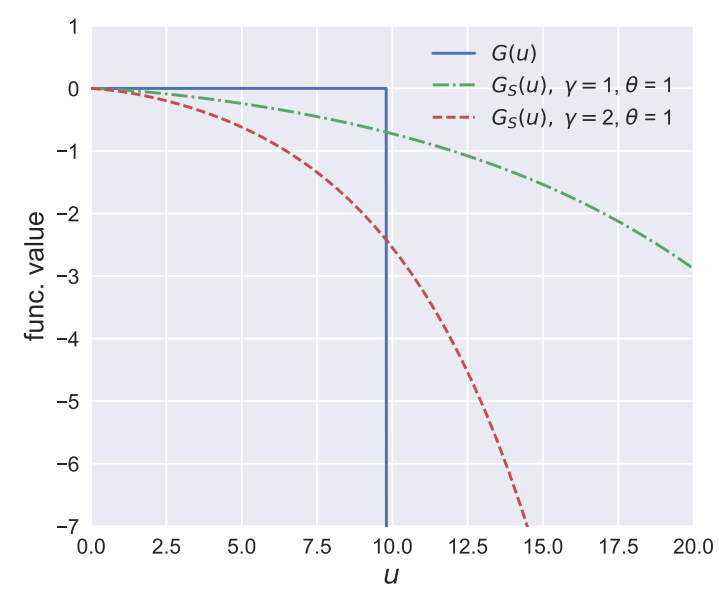

(b) Example of $G_{S}$ when $h(u)=1-\frac{1}{u+1}$

Figure 1: Examples of $G_{S}$ given in (14) for different values of $\gamma$ and $\theta$.

Example 3 (A-optimal experiment design). When $h(u)=1-\frac{1}{1+u}$, and so $h^{\prime}(u)=(1+u)^{-2}$, it is again possible to express the optimal $G_{S}^{\prime}$ in terms of exponential integrals. Using the same strategy as example 2, but with the bound $h^{\prime}(\theta v)=(1+\theta v)^{-2} \geq e^{-2 \theta v}$ (for $\left.v \geq 0\right)$, gives

$$
G_{S}^{\prime}(u) \leq \frac{1}{(e-1)} \frac{1-e^{\frac{\gamma}{b} u}}{\theta^{-1}+(2 b / \gamma)} \text { and } b^{\prime} \leq \frac{b}{\gamma} \log \left[(e-1) \Theta\left(\theta^{-1}+2 b / \gamma\right)+1\right] .
$$

Figure $1 b$ shows examples of $G_{S}$ for two values of $\gamma$ when $h(u)=1-\frac{1}{1+u}$.

\subsection{Smoothing $h$.}

We are now in a position to design $h_{S}$, a smoothed surrogate for $h$. Recall that, for a fixed choice of $\gamma \geq 1$, we can use (14) to design an optimal $G_{S}^{\prime}$. This gives a bound $b^{\prime}$ on the budget consumed by Algorithm 2. We design $h_{S}$ by solving the following optimization problem over $\beta$ and the function $h_{S}$ :

$$
\text { minimize } \beta \text { subject to }\left\{\begin{array}{l}
\gamma h_{S}(u) \leq h^{*}\left(h_{S}^{\prime}(u)\right)+\beta h(u) \quad \forall u \in\left[0, u_{\max }\right] \\
h_{S} \text { satisfies PSD-DR. }
\end{array}\right.
$$


Note that this problem comes directly from Lemma 3 and the fact that for fixed $\gamma$, we can maximize the bound on the competitive ratio, $(\gamma /(e-1)+\beta)^{-1}$, by minimizing $\beta$.

The key challenge in solving this optimization problem is imposing the PSD-DR property. A crucial observation in this paper is that, for a differentiable trace function, satisfying PSD-DR can be expressed in an equivalent, more explicit form. This is an easy consequence of Löwner's theorem for matrix monotone functions [Han13], an important result in matrix analysis.

Proposition 2. Suppose $H_{S}(U)=\sum_{i=1}^{n} h_{S}\left(\lambda_{i}(U)\right)$ where $h_{S}$ is a monotone increasing, concave function, and $h_{S}(0)=0$. Then $H_{S}$ is $P S D-D R$ for all $n$, if and only if there exists a positive measure $\mu$ supported on $[0,1]$ such that

$$
h_{S}(u)=\int_{0}^{u} y\left(u^{\prime}\right) d u^{\prime} \quad \text { where } \quad y(u)=\int_{0}^{1} \frac{1}{u \lambda+(1-\lambda)} d \mu(\lambda) .
$$

Proof. This follows from [Han13, Theorem 4.9].

This alternative description allows us to impose the constraint that $h_{S}$ satisfies PSD-DR in the optimization problem for designing $h_{S}$. We now rewrite this optimization problem in a more computationally useful form. We use the change of variable $h_{S}(u)=\int_{0}^{u} y(s) d s$, introduce $\mu$ from (17) as a decision variable, and express $y$ in terms of $\mu$. Doing so we obtain the following optimization problem.

$$
\begin{aligned}
& \operatorname{minimize}_{\beta, y, \mu} \beta \\
& \text { subject to } \gamma \int_{0}^{u} y(s) d s-h^{*}(y(u)) \leq \beta h(u) \quad \forall u \in\left[0, u_{\max }\right] \\
& y(t)=\int_{0}^{1} \frac{1}{t \lambda+(1-\lambda)} d \mu(\lambda) \\
& \mu \text { a positive measure supported on }[0,1] .
\end{aligned}
$$

We denote by $\beta(\gamma)$, the optimal value of (18) for a given $\gamma$. We extend $h_{S}$ linearly on $\mathbf{R}_{-}$to satisfy the technical assumption on the domain of $h_{S}$ in Assumption (1).

Theorem 1. Suppose that $G_{S}$ is defined as in (14), and $H_{S}$ is the trace function corresponding to $\int_{0}^{u} y(s) d s$, where $y$ is optimal for (18). Then the iterates $\tilde{x}_{1}, \ldots, \tilde{x}_{m}$ of Algorithm 2 satisfy

$$
H\left(\sum_{t=1}^{m} A_{t} \tilde{x}_{t}\right) \geq \frac{1}{\gamma /(e-1)+\beta(\gamma)} D^{\star} \quad \text { and } \quad \sum_{t=1}^{m} c_{t} \tilde{x}_{t} \leq G_{S}^{\prime-1}\left(-h^{\prime}(0) \Theta\right)
$$

where $\Theta \geq c_{t}^{-1} \operatorname{tr}\left(A_{t}\right)$ for all $t$ and $\beta(\gamma)$ is the optimal value of $\beta$ in (18). Moreover, if $H$ satisfies the PSD-DR property, then $\beta(\gamma) \leq \gamma+1$.

Proof. The proofs of the first two inequalities follow directly from Lemma 3. To see that $\beta(\gamma) \leq \gamma+1$ when $H$ satisfies the PSD-DR property, one can note that $y(u)=h^{\prime}(u)$ and $\beta=\gamma+1$ is a feasible solution for (18) in that case.

Note that if $H_{S}$ satisfies PSD-DR, then $H$ is a feasible solution to problem (18); however, it is not necessarily the optimal solution. As we will see in the next section, the objective function in D-optimal experiment design, $H(U)=\log \operatorname{det}(I+U)$, provides such and example, where the competitive ratio improves as the result of smoothing even though $H$ is PSD-DR. In the next section, we also consider the A-optimal experiment design where the objective function does not satisfy PSD-DR. 


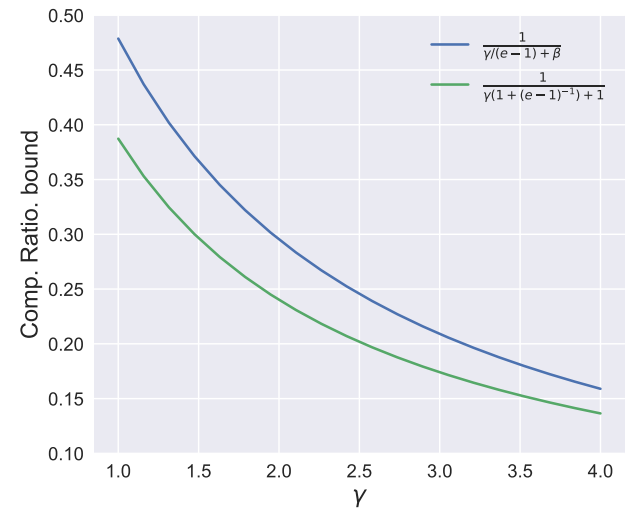

(a)

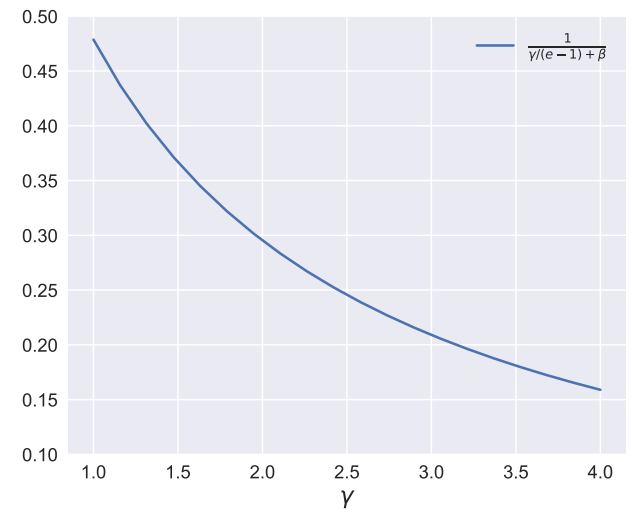

(b)

Figure 2: (a) Two competitive ratio bounds vs $\gamma$ for $h(u)=\log (u+1)$. The first bound is achieved by numerically finding the optimum $\beta(\lambda)$ in (16), while the second bound uses the fact that $\beta(\lambda) \leq \lambda+1$ when $h(u)=\log (u+1)$. (b) Competitive ratio bound achieved by numerically finding the optimum $\beta(\lambda)$ for $h(u)=1-1 /(u+1)$.

\subsubsection{Examples}

Example 4 (Linear objective). When $h(u)=u$ then

$$
h^{*}(y)= \begin{cases}0 & \text { if } y=1 \\ -\infty & \text { otherwise. }\end{cases}
$$

Therefore, the only feasible solution to (16) is $h_{S}(u)=u$. This is a PSD-DR function. The corresponding value of $\beta$ is $\beta=\gamma$. As such, the competitive ratio achieved by Algorithm 2 is at least $\frac{1}{\gamma}\left(1-\frac{1}{e}\right)$.

Example 5 (D-optimal experiment design). In this case $h(u)=\log (u+1)$, i.e., $H(U)=\log \operatorname{det}(I+$ $U)$. Note that $H$ satisfies the $P S D-D R$ property, because we can write $h^{\prime}(u)=1 /(1+u)$ in the form of (17) by choosing the measure $\mu(\lambda)$ to have mass $1 / 2$ at $\lambda=1 / 2$. By putting $h_{S}(u)=h(u)$, we can conclude that $\beta(\gamma) \leq 1+\gamma$. This means that the competitive ratio achieved by Algorithm 2 is at least $\frac{1}{\gamma(1-1 / e)^{-1}+1}$.

By solving (18) computationally, we can design an $h_{S}$ that achieves a better competitive ratio. Figure $3 a$ shows the solution of (18) for $\gamma=1$ over the finite horizon $\left[0, u_{\max }\right]=[0,1]$. Figures $3 b$ and $3 c$ show the solution of (18) for $\gamma=4$ and $u_{\max }=1$ and $u_{\max }=10$ respectively. We note that the optimal measures are quite complicated - in the case $\gamma=1$ the optimal measure seems to be atomic, whereas in the case $\gamma=4$, it has a qualitatively different structure. In Figure 2a, we plot the competitive ratio bound of Theorem 1 vs $\gamma$, and compare it with the bound obtained without smoothing $h$. We can see that $\gamma$ captures the trade off between the budget violation and competitive ratio. A smaller $\gamma$ gives a better competitive ratio at the expense of larger budget violation, quantified by the bound given in Example 2.

Example 6 (A-optimal experiment design.). In this case $H$ is not a PSD-DR function, so to obtain competitive ratio bounds by our method we must construct a PSD-DR surrogate $H_{S}$ numerically. Figure 2b, shows the competitive ratio bound given by solving (18) for $h(u)=1-1 /(u+1)$ for different values of the parameter $\gamma$. In Figure 4, we have provided examples of $h_{S}$ for different values of $\gamma$ and $u_{\max }$. 

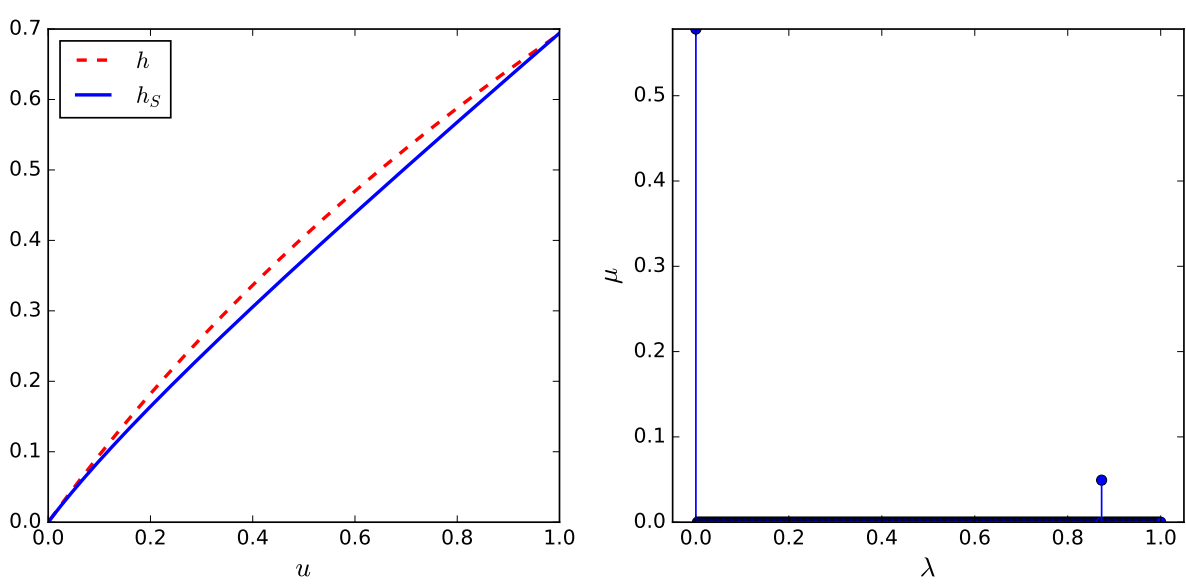

(a)
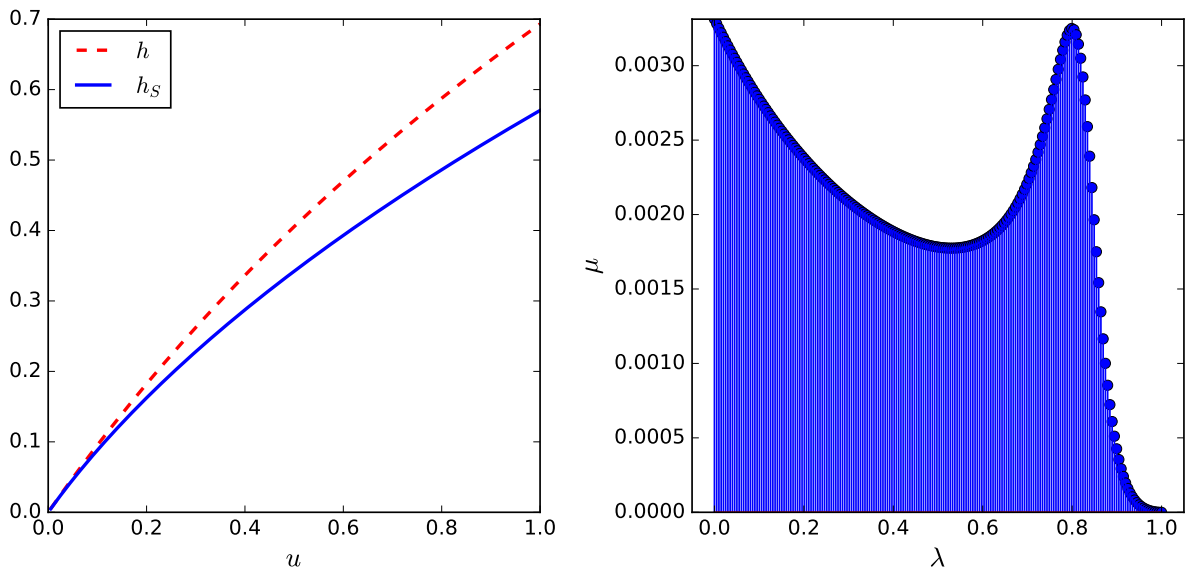

(b)
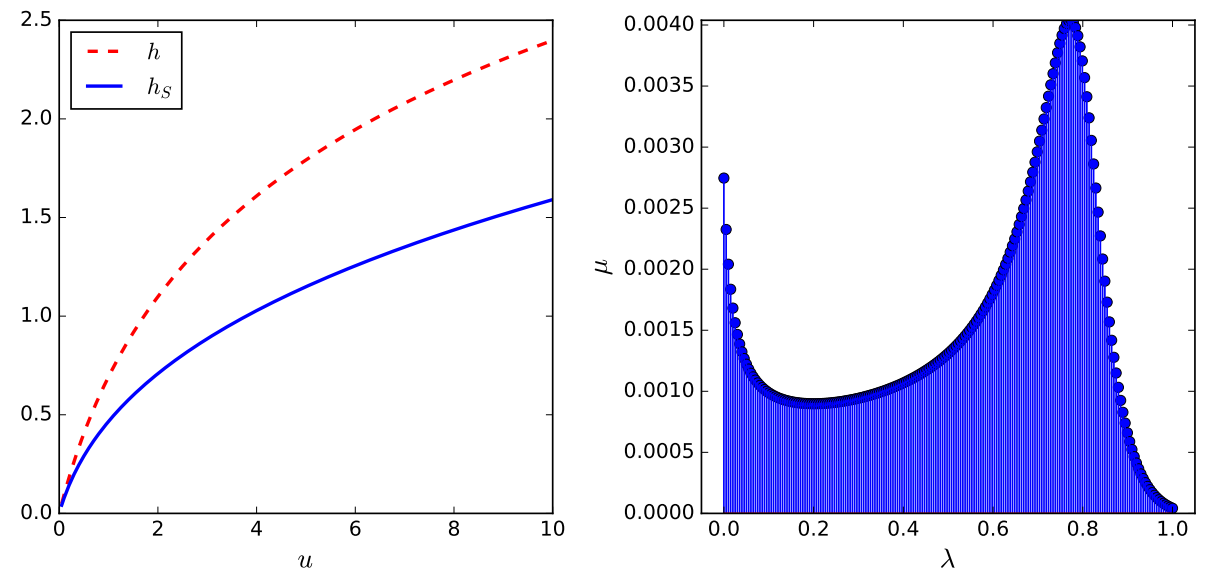

(c)

Figure 3: The smoothed function $h_{S}$ and the corresponding measure $\mu$, when $h(u)=\log (u+1)$. The smooth $h_{S}$ is found by solving the convex problem (18) via discretization of measure $\mu$. In (a), $\gamma=1$ and $u_{\max }=1$. In (b), $\gamma=4$ and $u_{\max }=1$. In (c), $\gamma=4$ and $u_{\max }=10$. 

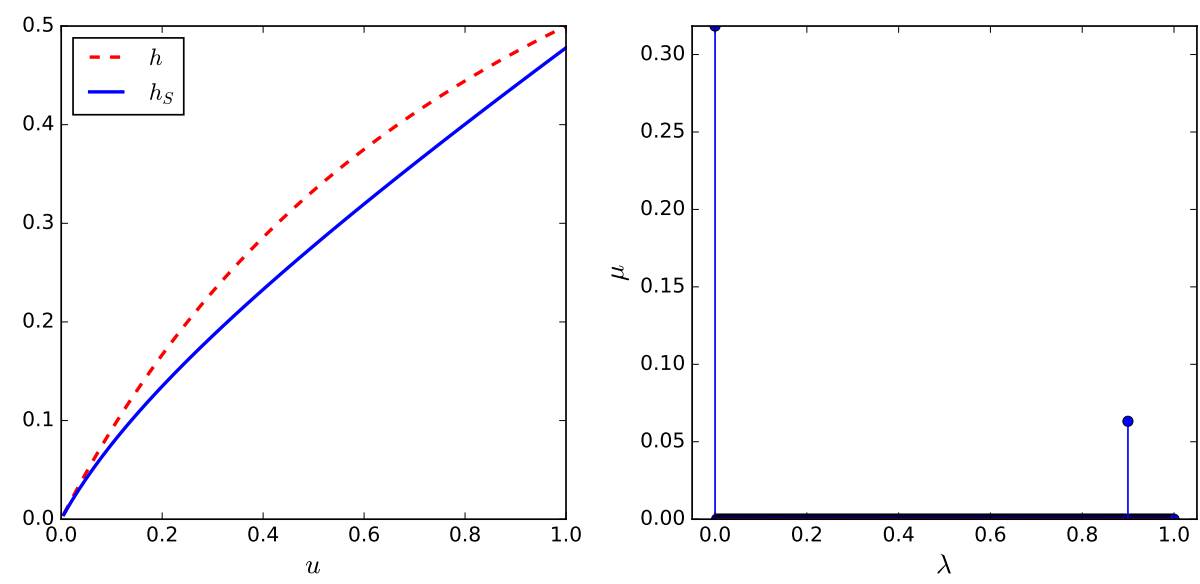

(a)
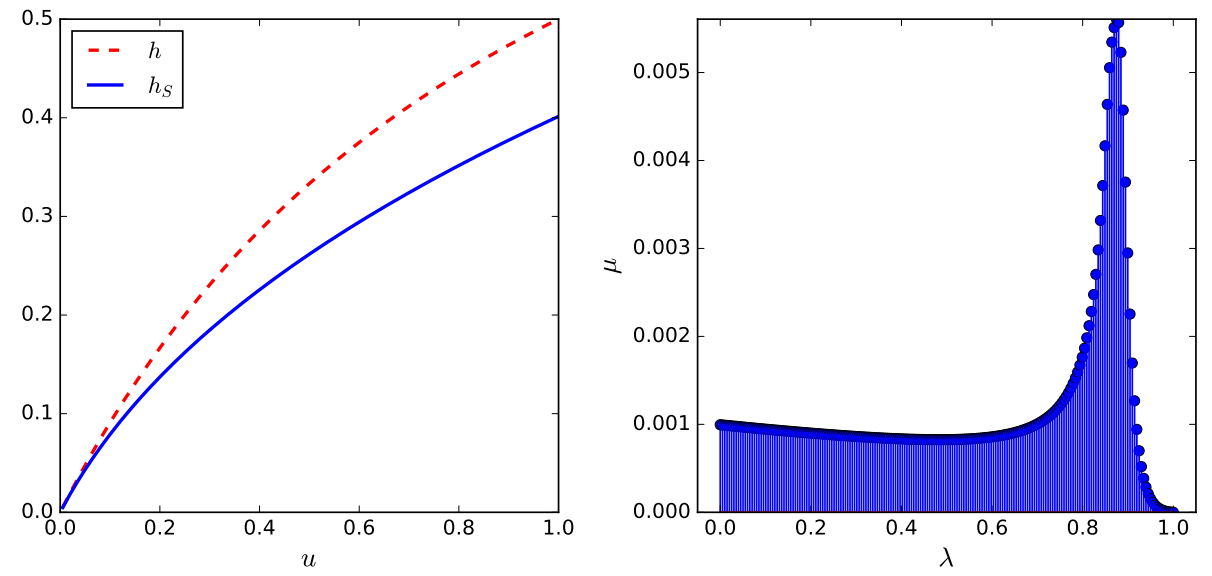

(b)
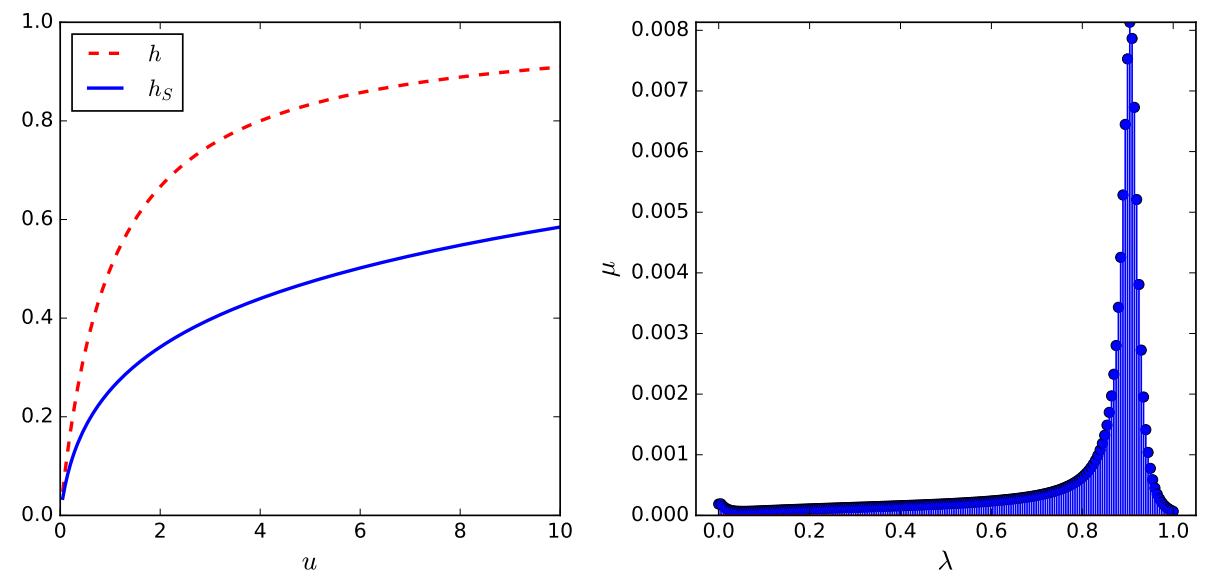

(c)

Figure 4: The smoothed function $h_{S}$ and the corresponding measure $\mu$, when $h(u)=1-\frac{1}{u+1}$. The smooth $h_{S}$ is found by solving the convex problem (18) via discretization of measure $\mu$. In (a), $\gamma=1$ and $u_{\max }=1$. In (b), $\gamma=4$ and $u_{\max }=1$. In (c), $\gamma=4$ and $u_{\max }=10$. 


\section{Analysis of the sequential algorithm}

To design the functions $H_{S}$ and $G_{S}$ for the sequential algorithm (Algorithm 1), we need additional information about the problem data. Since Algorithm 1 uses the dual variable from the previous time step to assign the primal variable, the maximum length of each primal step, which is captured by the following two parameters, plays a role in the competitive ratio. Let

$$
\rho_{1} \geq \max _{t} c_{t}, \quad \text { and } \quad \rho_{2} \geq \max _{t} \lambda_{\max }\left(A_{t}\right) .
$$

The next lemma is an analogue of Lemma 3 but for the sequential algorithm.

Lemma 4. Let $G_{S}$ satisfy Assumption 2, $h_{S}$ satisfy Assumption 1, and suppose that the corresponding trace function $H_{S}$ satisfies the PSD-DR assumption. Suppose that $\theta \leq c_{t}^{-1} \operatorname{tr}\left(A_{t}\right) \leq \Theta$ for all $t$, that $\rho_{1}$ and $\rho_{2}$ are defined as in (19), and $u_{\max } \geq \lambda_{\max }\left(\sum_{t=1}^{m} A_{t} \hat{x}_{t}\right) .{ }^{4}$ Then

1. If for a given $\gamma \geq 1, G_{S}$ satisfies:

$$
\gamma\left[G_{S}(u)-\rho_{1} G_{S}^{\prime}(u)\right] \leq G^{*}\left(G_{S}^{\prime}(u)\right)+\frac{\gamma}{e-1} h(\theta u) \quad \forall u \in[0, \infty)
$$

and there exists $\beta>0$ such that $h_{S}$ satisfy the following inequlity:

$$
\gamma\left[h_{S}(u)+\rho_{2}\left(h_{S}^{\prime}(0)-h_{S}^{\prime}(u)\right)\right] \leq h^{*}\left(h_{S}^{\prime}(u)\right)+\beta h(u) \quad \forall u \in\left[0, u_{\max }\right]
$$

then the iterates $\hat{x}_{1}, \ldots, \hat{x}_{m}$ of Algorithm 1 satisfy

$$
H\left(\sum_{t=1}^{m} A_{t} \hat{x}_{t}\right) \geq \frac{1}{\gamma /(e-1)+\beta} D^{\star} .
$$

2. The iterates $\hat{x}_{1}, \ldots, \hat{x}_{m}$ of Algorithm 1 satisfy

$$
\sum_{t=1}^{m} c_{t} \hat{x}_{t} \leq b^{\prime} \quad \text { where } \quad b^{\prime}:=\rho_{1}+\inf \left\{u: G_{S}^{\prime}(u) \leq-h^{\prime}(0) \Theta\right\} .
$$

Proof. First we show that $\sum_{t=1}^{m} c_{t} \hat{x}_{t} \leq b^{\prime}$. Arguing by contradiction, let $T$ be the smallest index such that $\sum_{t=1}^{T+1} c_{t} \hat{x}_{t}>b^{\prime}$. Then, $\sum_{t=1}^{T} c_{t} \hat{x}_{t}>b^{\prime}-\rho_{1}\left(\right.$ since $\left.c_{T+1} \hat{x}_{T+1} \leq \rho_{1}\right)$. We have that $\hat{z}_{T}=G_{S}^{\prime}\left(\sum_{t=1}^{T} c_{t} \hat{x}_{t}\right)<-h^{\prime}(0) \Theta$, where the inequality holds by the definition of $b^{\prime}$. By the PSDDR assumption, $\nabla H_{S}\left(\sum_{t=1}^{T} A_{t} \hat{x}_{t}\right) \preceq \nabla H_{S}(0)=h_{S}^{\prime}(0) I$. Then

$$
\left\langle\hat{Y}_{T}, A_{T+1}\right\rangle+c_{T+1} \hat{z}_{T} \leq c_{T+1}\left(h_{S}^{\prime}(0) c_{T+1}^{-1} \operatorname{tr}\left(A_{T+1}\right)+\hat{z}_{T}\right)<0 .
$$

It follows that $\hat{x}_{T+1}=0$, contradicting our choice of $T$. Hence $\sum_{t=1}^{m} c_{t} \hat{x}_{t} \leq b^{\prime}$.

We now bound on the competitive ratio. Let $U=\sum_{t=1}^{m} A_{t} \hat{x}_{t}, u=\sum_{t=1}^{m} c_{t} \hat{x}_{t}, \hat{Y}_{m}=\nabla H_{S}(U)$, and $\hat{z}_{m}=G_{S}^{\prime}(u)$. First we note that

$$
\begin{aligned}
\sum_{t=1}^{m}\left[\left\langle A_{t} \hat{x}_{t}, \hat{Y}_{t-1}-\hat{Y}_{t}\right\rangle+c_{t} \hat{x}_{t}\left(\hat{z}_{t-1}-\hat{z}_{t}\right)\right] & \leq \sum_{t=1}^{m}\left[\rho_{2} \operatorname{tr}\left(\hat{Y}_{t-1}-\hat{Y}_{t}\right)+\rho_{1}\left(\hat{z}_{t-1}-\hat{z}_{t}\right)\right] \\
& =\rho_{2}\left(\operatorname{tr}\left(\hat{Y}_{0}-\hat{Y}_{m}\right)\right)-\rho_{1}\left(\hat{z}_{m}\right)
\end{aligned}
$$

\footnotetext{
${ }^{4}$ Note that we could choose, for instance, $u_{\max }=b^{\prime} \max _{t} c_{t}^{-1} \lambda_{\max }\left(A_{t}\right)$, but for certain classes of problems better bounds may be available.
} 
where the inequality holds because $\hat{Y}_{t} \preceq \hat{Y}_{t-1}$ (by the PSD-DR assumption), and, similarly, $\hat{z}_{m} \leq \cdots \leq \hat{z}_{0}=0$ (since $G_{S}^{\prime}(0)=0$ ). By the primal allocation rule in Algorithm 1, we have $\hat{x}_{t}\left(c_{t} \hat{z}_{t-1}+\left\langle A_{t}, \hat{Y}_{t-1}\right\rangle\right) \geq 0$. Combining this with the concavity of $H_{S}$ and $G_{S}$, we get

$$
\begin{array}{r}
H_{S}\left(\sum_{s=1}^{t} A_{s} \hat{x}_{s}\right)+G_{S}\left(\sum_{s=1}^{t} c_{s} \hat{x}_{s}\right)-H_{S}\left(\sum_{s=1}^{t-1} A_{s} \hat{x}_{s}\right)-G_{S}\left(\sum_{s=1}^{t-1} c_{s} \hat{x}_{s}\right) \\
+\hat{x}_{t}\left(c_{t}\left(\hat{z}_{t-1}-\hat{z}_{t}\right)+\left\langle A_{t}, \hat{Y}_{t-1}-\hat{Y}_{t}\right\rangle\right) \geq 0
\end{array}
$$

Taking the sum over $t$, telescoping, and using $h_{S}(0)=G_{S}(0)=0$, gives

$$
H_{S}(U)+G_{S}(u)+\sum_{t=1}^{m}\left[\left\langle A_{t} \hat{x}_{t}, \hat{Y}_{t-1}-\hat{Y}_{t}\right\rangle+c_{t} \hat{x}_{t}\left(\hat{z}_{t-1}-\hat{z}_{t}\right)\right] \geq 0 .
$$

Now, the proof follows the same step as the proof of Lemma 3 and uses (6) and the above inequalities.

$$
\begin{aligned}
& H(U)-D_{\text {seq }} \\
& \geq H(U)+H^{*}\left(\hat{Y}_{m}\right)-H_{S}(U)-G_{S}(u)+G^{*}\left(\hat{z}_{m}\right) \\
& +\sum_{t=1}^{m} \hat{x}_{t}\left[\left\langle A_{t}, \hat{Y}_{t}-\hat{Y}_{t-1}\right\rangle+c_{t}\left(\hat{z}_{t}-\hat{z}_{t-1}\right)\right] \\
& \geq\left[1-\frac{\gamma}{e-1}\right] H(U)+H^{*}\left(\hat{Y}_{m}\right)-H_{S}(U)-(1-\gamma) G_{S}(u)-\gamma \rho_{1} G_{S}^{\prime}(u) \\
& +(\gamma+(1-\gamma)) \sum_{t=1}^{m} \hat{x}_{t}\left[\left\langle A_{t}, \hat{Y}_{t}-\hat{Y}_{t-1}\right\rangle+c_{t}\left(\hat{z}_{t}-\hat{z}_{t-1}\right)\right] \quad \text { By (20) and (13) } \\
& \geq\left[1-\frac{\gamma}{e-1}\right] H(U)+H^{*}\left(\hat{Y}_{m}\right)-H_{S}(U)+(1-\gamma) H_{S}(U)-\gamma \rho_{1} \hat{z}_{m} \\
& +\gamma \sum_{t=1}^{m} \hat{x}_{t}\left[\left\langle A_{t}, \hat{Y}_{t}-\hat{Y}_{t-1}\right\rangle+c_{t}\left(\hat{z}_{t}-\hat{z}_{t-1}\right)\right] \\
& \geq\left[1-\frac{\gamma}{e-1}\right] H(U)+H^{*}\left(\hat{Y}_{m}\right)-\gamma\left[H_{S}(U)+\rho_{2} \operatorname{tr}\left(\hat{Y}_{0}-\hat{Y}_{m}\right)\right] \\
& \geq\left[1-\frac{\gamma}{e-1}-\beta\right] H(U)
\end{aligned}
$$

Applying Lemma 2 completes the proof.

For the sequential algorithm to minimize the bound $b^{\prime}$ on the budget consumption we choose $G_{S}$ such that

$$
G_{S}^{\prime}(u)=-\frac{\theta \gamma}{\left(b+\rho_{1} \gamma\right)(e-1)} \int_{0}^{u} \exp \left(\frac{\gamma}{b+\rho_{1} \gamma}(u-v)\right) h^{\prime}(\theta v) d v
$$

To find $h_{S}$ for the sequential algorithm, the problem (18) is modified to:

$$
\begin{aligned}
& \operatorname{minimize}_{\beta, y, \mu} \beta \\
& \text { subject to } \gamma \int_{0}^{u} y(s) d s+\gamma \rho_{2}(y(0)-y(u))-h^{*}(y(u)) \leq \beta h(u) \quad \forall u \in\left[0, u_{\text {max }}\right] \\
& y(t)=\int_{0}^{1} \frac{1}{t \lambda+(1-\lambda)} d \mu(\lambda) \\
& \mu \text { a positive measure supported on }[0,1] .
\end{aligned}
$$



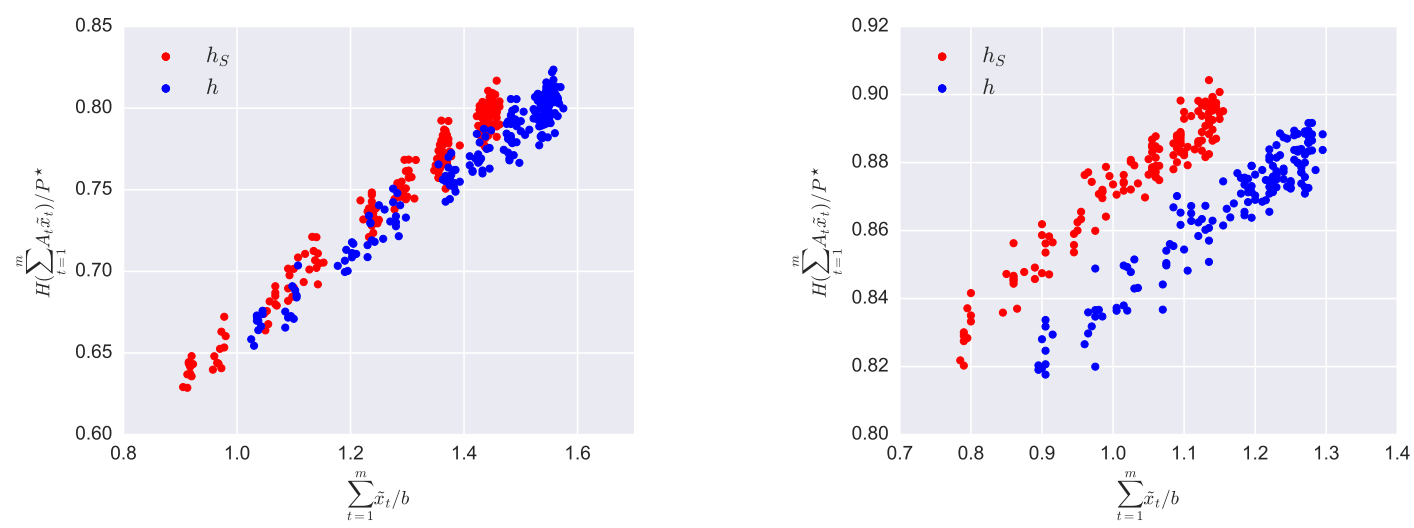

Figure 5: Performance of Algorithm 2 (sequential updates) for online D-optimal and A-optimal experiment design with two choices of $H_{S}$. (Left) D-optimal experiment design. (Right) A-optimal experiment design.

Let $\beta(\gamma)$ be the optimal value of $\beta$ in (27). Combining our arguments gives the following analogue of Theorem 1 for the sequential algorithm. It describes the tradeoff between budget consumption and competitive ratio achieved for the sequential algorithm when $G_{S}$ and $H_{S}$ are designed optimally.

Theorem 2. If $G_{S}$ is defined by (26), and $H_{S}$ is the trace function corresponding to $\int_{0}^{u} y(s) d s$, where $y$ is optimal for (27), then the iterates, $\hat{x}_{1}, \ldots, \hat{x}_{m}$ of Algorithm 1 satisfy

$$
\sum_{t=1}^{m} c_{t} \hat{x}_{t} \leq G_{S}^{\prime-1}\left(-h^{\prime}(0) \Theta\right)+\rho_{1} \quad \text { and } \quad H\left(\sum_{t=1}^{m} A_{t} \hat{x}_{t}\right) \geq \frac{1}{\gamma /(e-1)+\beta(\gamma)} D^{\star}
$$

where $c_{t}^{-1} \operatorname{tr}\left(A_{t}\right) \leq \Theta$ for all $t$.

Proof. The proof is the natural analogue of Theorem 1.

\section{$5 \quad$ Numerical Experiments}

In this section, we provide the results of our numerical experiments and discuss the numerical implementation of smoothing design.

In our numerical simulations, we consider the D-optimal $(H(U)=\log \operatorname{det}(I+U))$ and the A-optimal $\left(H(U)=n-\operatorname{tr}\left((I+U)^{-1}\right)\right)$ experiment design problems. The $t^{t h}$ matrix $A_{t}=a_{t} a_{t}^{T}$ is generated as follows: we sample a vector $\eta$ uniformly at random from $\{-1,1\}^{n}$ and then set $a_{t}=\frac{\sqrt{m-t+1}}{\sqrt{n}} \eta$. This is an adversarial weighting, inspired by worst-case examples for online LP, e.g., [BN09b].

We compare the performance of Algorithm 2 (sequential updates) with two choices of $H_{S}$. In one case, we use the smoothed $H_{S}$ given by solving (18). In the second case, we use $H$ without smoothing $\left(H_{S}=H\right)$. For both cases, we use the smooth $G_{S}$ given in (14). (Note that a pure greedy algorithm without smoothing $G$ simply picks the first $b$ experiments and is a trivial algorithm, which we did not try.) We varied the parameter $\gamma$ and plotted the competitive ratio versus the budget used by the algorithm in Figure 5. For each value of $\gamma$, we have 10 random repeats.

Next, we briefly discuss the numerical implementation of the smoothing design problems for the simultaneous algorithm, introduced in Section 3. Similar ideas apply for the smoothing design 
problems for the sequential algorithm. We note that the algorithm does not require $G_{S}$ itself, but only $G_{S}^{\prime}(u)$. The formula for $G_{S}^{\prime}$ can be computed up to desired accuracy using Gauss-Leguerre quadrature [Rei43].

To solve problem (18), we restrict $\mu$ to be an atomic measure supported on the $q+1$ points $\lambda_{j}=j / q \in[0,1]$ for $j=0,1, \ldots, q$. The decision variables are then $\beta$ and $\mu_{j}:=\mu\left(\lambda_{j}\right)$ for $j=0,1, \ldots, q$. Rather than imposing the constraint for all $u \in\left[0, u_{\max }\right]$ we impose it on a non-uniformly sampled subset. In particular, we sample $u$ more densely where $h$ has a larger local Lipschitz constant by choosing the discretization points to be $u_{i}=h^{-1}\left(i u_{\max } / d\right)$ for $i=0,1, \ldots, d$. The optimal $y=h_{S}^{\prime}$, which is all that is needed for the algorithm, is $y(u)=\sum_{j=0}^{q} \frac{\mu_{j}}{u \lambda_{j}+\left(1-\lambda_{j}\right)}$. Note that since the integral of every individual function in the summand representation of $h_{S}^{\prime}$ satisfies PSD-DR, $h_{S}$ satisfies PSD-DR.

\section{Related Work and Discussion}

Submodularity and experiment design. Algorithms for the offline optimal experiment design (both with and without integer constraints) have been extensively studied [Puk93]. Let $X_{S}$ denote a principal submatrix of $X$, then it is well known that the set function $S \mapsto \log \operatorname{det}\left(X_{S}\right)$ is submodular. Based on this, greedy subset selection is shown to have a $1-1 / e$ approximation ratio [BGS10, SBV10, See09] for the (integer) experiment selection problem. The paper [BBKT17] also gives an approximation ratio for the weakly submodular A-optimal design problem; also see [WYS16] and references therein for statistical bounds for solving the convex relaxation followed by a greedy postprocessing. In the existing literature, however, algorithms are assumed to have access to all possible experiments at the start, and therefore the result do not apply to the worst-case online setting that we consider. There is a connection between analysis of online algorithm under stochastic i.i.d setting and greedy algorithm for submodular maximization. We refer the reader to [Egh17] for the details of this connection.

Online SDP problem of [EKN16]. To the best of our knowledge, [EKN16] is the only existing work that studies an online semidefinite program. While the problem considered is different from ours and its results do not apply to our setup, we briefly discuss the idea. [EKN16] considers a generalization of the online covering linear program to the semidefinite cone as follows,

$$
\text { minimize } b^{T} y \text { subject to }\left\{\begin{array}{l}
\mathcal{A}(y) \succeq C \\
y \geq 0
\end{array}\right.
$$

where $\mathcal{A}$ denotes a linear map from vectors to matrices. The algorithm receives a sequence of PSD matrices $C^{(0)} \preceq C^{(1)} \preceq \ldots \preceq C^{(m)}=C$ over time, and needs to increase the variable $y$ so as to satisfy the new matrix covering constraint. The dual of this problem is a packing problem, however the model for the online information the algorithm receives is still the sequence of matrices $C^{(i)}$ which is different from receiving a new experiment and bid price to decide on, as in our model. Indeed, extending the packing linear program to the PSD cone yields a different problem, i.e.,

$$
\text { maximize } c^{T} x \quad \text { subject to }\left\{\begin{array}{l}
\mathcal{A}(x) \preceq B \\
x \geq 0,
\end{array}\right.
$$

which is in line with the setting we consider in this paper, after a reformulation to bring the matrix constraint to the objective in a penalized form (which corresponds to $H$ ). The possibility of using Löwner's representation to design a matrix penalty function for this matrix-valued budget is an interesting direction for future work. 
Online Learning, Regret, and FTRL. As mentioned earlier, the dual update in Algorithm 1 is the same as in Follow-the-Regularized-Leader (FTRL) algorithm with $-H_{S}{ }^{*}$ as the regularizer. This primal-dual perspective has been used in [SSS07] for design and analysis of online learning algorithms. In the online learning literature, the goal is to derive a bound on the regret that optimally depends on the horizon, $m$; whereas in this work we study the competitive ratio for the algorithm that depends on the functions $H$ and $G$. In order to optimize the competitive ratio, the regularization functions should be crafted based on $H$, and a general choice of regularization which yields an optimal regret bound in terms of $m$ is not enough for a competitive ratio argument, so existing results in online learning do not address our aim. There are however some shared proof steps in the analysis that are worth exploring further.

\section{Acknowledgements}

The authors thank Omid Sadeghi-Meibodi for helpful comments. The work of MF and RE was supported in part by grants ONR N000141612789, NSF CCF 1409836, NSF Tripods 1740551, and ONR MURI N000141612710. Part of this work was done while RE and MF were visiting the Simons Institute for the Theory of Computing, partially supported by the DIMACS/Simons Collaboration on Bridging Continuous and Discrete Optimization through NSF grant CCF-1740425.

\section{References}

$\left[\mathrm{ABC}^{+} 16\right]$ Y. Azar, N. Buchbinder, TH H. Chan, S. Chen, I. R. Cohen, A. Gupta, Z. Huang, N. Kang, V. Nagarajan, J. Naor, et al. Online algorithms for covering and packing problems with convex objectives. In Foundations of Computer Science (FOCS), 2016 IEEE 57th Annual Symposium on, pages 148-157. IEEE, 2016. 1

[AD15] S. Agrawal and N. R. Devanur. Fast algorithms for online stochastic convex programming. In Proceedings of the Twenty-Sixth Annual Symposium on Discrete Algorithms, pages 1405-1424. SIAM, 2015. 2

[AWY14] Shipra Agrawal, Zizhuo Wang, and Yinyu Ye. A dynamic near-optimal algorithm for online linear programming. Operations Research, 62(4), 2014. 1

[BBKT17] Andrew An Bian, Joachim M Buhmann, Andreas Krause, and Sebastian Tschiatschek. Guarantees for greedy maximization of non-submodular functions with applications. arXiv preprint arXiv:1703.02100, 2017. 19

[BGS10] M. Bouhtou, S. Gaubert, and G. Sagnol. Submodularity and randomized rounding techniques for optimal experimental design. Electronic Notes in Discrete Mathematics, 36:679-686, 2010. 19

[BJN07] Niv Buchbinder, Kamal Jain, and Joseph Seffi Naor. Online primal-dual algorithms for maximizing ad-auctions revenue. In Algorithms-ESA 200\%, pages 253-264. Springer, 2007. 4

[BN06] Niv Buchbinder and Joseph Naor. Improved bounds for online routing and packing via a primal-dual approach. In 47th Annual IEEE Symposium on Foundations of Computer Science (FOCS'06), pages 293-304. IEEE, 2006. 1

[BN09a] Niv Buchbinder and Joseph Naor. Online primal-dual algorithms for covering and packing. Mathematics of Operations Research, 34(2):270-286, 2009. 4, 7, 10 
[BN09b] Niv Buchbinder and Joseph Seffi Naor. The design of competitive online algorithms via a primal-dual approach. Foundations and Trends® in Theoretical Computer Science, 3(2-3):93-263, 2009. 1, 2, 18

[DJ12] Nikhil R Devanur and Kamal Jain. Online matching with concave returns. In Proceedings of the forty-fourth annual ACM symposium on theory of computing (STOC), pages 137-144. ACM, 2012. 4

[Dra03] Sever S Dragomir. Some Gronwall type inequalities and applications. Nova Science, 2003. 10

[EF16a] Reza Eghbali and Maryam Fazel. Designing smoothing functions for improved worstcase competitive ratio in online optimization. In Advances in Neural Information Processing Systems, pages 3279-3287, 2016. 3, 4, 7, 10

[EF16b] Reza Eghbali and Maryam Fazel. Worst case competitive analysis of online algorithms for conic optimization. arXiv preprint arXiv:1611.0050\%, 2016. 6

[Egh17] Reza Eghbali. Online algorithm design via smoothing with application to online experiment selection. PhD thesis, The University of Washington, 8 2017. 19

[EKN16] Noa Elad, Satyen Kale, and Joseph Seffi Naor. Online semidefinite programming. In LIPIcs-Leibniz International Proceedings in Informatics, volume 55. Schloss DagstuhlLeibniz-Zentrum fuer Informatik, 2016. 19

[ESF14] Reza Eghbali, Jon Swenson, and Maryam Fazel. Exponentiated subgradient algorithm for online optimization under the random permutation model. arXiv preprint arXiv:1410.7171, 2014. 2

[GM16] Anupam Gupta and Marco Molinaro. How the experts algorithm can help solve lps online. Mathematics of Operations Research, 41(4):1404-1431, 2016. 2

[Han13] Frank Hansen. The fast track to Löwners theorem. Linear Algebra and its Applications, 438(11):4557-4571, 2013. 12

[KPV13] M. Kapralov, I. Post, and J. Vondrák. Online submodular welfare maximization: Greedy is optimal. In Proceedings of the Twenty-Fourth Annual Symposium on Discrete Algorithms, pages 1216-1225. SIAM, 2013. 1

[Lew95] Adrian S Lewis. The convex analysis of unitarily invariant matrix functions. Journal of Convex Analysis, 2(1):173-183, 1995. 4

[LJ16] Antoine Legrain and Patrick Jaillet. A stochastic algorithm for online bipartite resource allocation problems. Computers \& Operations Research, 75:28-37, 2016. 2

[MFM16] De Meng, Maryam Fazel, and Mehhran Mesbahi. Online algorithms for network formation. In IEEE Conference on Decision and Control (CDC), pages 135-140, Dec 2016. 2

[MR13] Marco Molinaro and R Ravi. The geometry of online packing linear programs. Mathematics of Operations Research, 39(1):46-59, 2013. 2

[MSVV07] Aranyak Mehta, Amin Saberi, Umesh Vazirani, and Vijay Vazirani. Adwords and generalized online matching. Journal of the ACM, 54(5):22, 2007. 1 
[Puk93] Friedrich Pukelsheim. Optimal design of experiments, volume 50. SIAM, 1993. 19

[Rei43] Anders Reiz. On the numerical solution of certain types of integral equations. Meddelanden fran Lunds Astronomiska Observatorium Serie I, 161:1-21, 1943. 19

[RWW98] R Tyrrell Rockafellar, Roger J-B Wets, and Maria Wets. Variational analysis, volume 317. Springer, 1998. 5

[SBV10] Manohar Shamaiah, Siddhartha Banerjee, and Haris Vikalo. Greedy sensor selection: Leveraging submodularity. In 49th IEEE Conference on Decision and Control (CDC), pages 2572-2577. IEEE, 2010. 19

[See09] Matthias Seeger. On the submodularity of linear experimental design. Technical report, Saarland University, 2009. 19

[SSS07] Shai Shalev-Shwartz and Yoram Singer. A primal-dual perspective of online learning algorithms. Machine Learning, 69(2-3):115-142, 2007. 20

[WYS16] Yining Wang, Adams Wei Yu, and Aarti Singh. On computationally tractable selection of experiments in regression models. arXiv preprint arXiv:1601.02068, 2016. 19

\section{A Additional proofs}

Here, we provide additional proofs not given in detail in the body of the paper.

Proof of Lemma 1. By the definition of $D_{\text {sim }}$, the definition of $\tilde{x}_{t}$, and the concavity of $H_{S}$ and $G_{S}$, we have that

$$
\begin{aligned}
D_{\text {sim }}= & \sum_{t=1}^{m}\left[\left\langle A_{t} \tilde{x}_{t}, \tilde{Y}_{t}\right\rangle+c_{t} \tilde{x}_{t} \tilde{z}_{t}\right]-H^{*}\left(\tilde{Y}_{m}\right)-G^{*}\left(\tilde{z}_{m}\right) \\
\leq \sum_{t=1}^{m}[ & {\left[H_{S}\left(\sum_{s=1}^{t} A_{s} \tilde{x}_{s}\right)-H_{S}\left(\sum_{s=1}^{t-1} A_{s} \tilde{x}_{s}\right)\right.} \\
& \left.\quad+G_{S}\left(\sum_{s=1}^{t} c_{s} \tilde{x}_{s}\right)-G_{S}\left(\sum_{s=1}^{t-1} c_{s} \tilde{x}_{s}\right)\right]-H^{*}\left(\tilde{Y}_{m}\right)-G^{*}\left(\tilde{z}_{m}\right) \\
= & H_{S}\left(\sum_{s=1}^{m} A_{s} \tilde{x}_{s}\right)+G_{S}\left(\sum_{s=1}^{m} c_{s} \tilde{x}_{s}\right)-H^{*}\left(\tilde{Y}_{m}\right)-G^{*}\left(\tilde{z}_{m}\right) .
\end{aligned}
$$

The inequality follows from concavity of $G_{S}$ and $H_{S}$. The final equality holds by telescoping the sum and using the fact that $H_{S}(0)=0=G_{S}(0)$. For the sequential algorithm we can write:

$$
\begin{aligned}
D_{\text {seq }} & =\sum_{t=1}^{m}\left[\left\langle A_{t} \hat{x}_{t}, \hat{Y}_{t-1}\right\rangle+c_{t} \hat{x}_{t} \hat{z}_{t-1}\right]-H^{*}\left(\hat{Y}_{m}\right)-G^{*}\left(\hat{z}_{m}\right) \\
& =\sum_{t=1}^{m}\left[\left\langle A_{t} \hat{x}_{t}, \hat{Y}_{t}\right\rangle+c_{t} \hat{x}_{t} \hat{z}_{t}\right]-H^{*}\left(\hat{Y}_{m}\right)-G^{*}\left(\hat{z}_{m}\right)+\sum_{t=1}^{m}\left[\left\langle A_{t} \hat{x}_{t}, \hat{Y}_{t-1}-\hat{Y}_{t}\right\rangle+c_{t} \hat{x}_{t}\left(\hat{z}_{t-1}-\hat{z}_{t}\right)\right]
\end{aligned}
$$

Now, the rest follows similar to steps as the simultaneous case.

Proof of Lemma 2. We write out the argument for the inequality $D_{\text {sim }} \geq D^{\star}$. The argument showing that $D_{\text {seq }} \geq D^{\star}$ is identical. We first show that the PSD-DR assumption on $H_{S}$ implies

$$
\sum_{t=1}^{m}\left(\left\langle A_{t}, \tilde{Y}_{t}\right\rangle+c_{t} \tilde{z}_{t}\right)_{+} \geq \sum_{s=1}^{m}\left(\left\langle A_{s}, \tilde{Y}_{m}\right\rangle+c_{s} \tilde{z}_{m}\right)_{+} .
$$


Since $A_{s} \in S_{+}^{n}$ and $\tilde{x}_{s} \geq 0$ for all $s \in[m]$, it follows that $\sum_{s=1}^{t} A_{s} \tilde{x}_{s} \preceq \sum_{s=1}^{m} A_{s} \tilde{x}_{s}$ for all $t \in[m]$. Since $\tilde{Y}_{t}=\nabla H_{S}\left(\sum_{s=1}^{t} A_{s} \tilde{x}_{s}\right)$, if $H_{S}$ satisfies the PSD-DR assumption then $\tilde{Y}_{t} \succeq \tilde{Y}_{m}$ for all $t \in[\mathrm{m}]$. By a similar argument, since $G_{S}$ is concave, $\tilde{z}_{t} \geq \tilde{z}_{m}$ for all $t \in[m]$. Since $A_{t} \in S_{+}^{n}$ and $c_{t} \geq 0$ for all $t \in[m]$,

$$
\left\langle A_{t}, \tilde{Y}_{t}\right\rangle+c_{t} \tilde{z}_{t} \geq\left\langle A_{t}, \tilde{Y}_{m}\right\rangle+c_{t} \tilde{z}_{t} \geq\left\langle A_{t}, \tilde{Y}_{m}\right\rangle+c_{t} \tilde{z}_{m}
$$

for all $t \in[m]$. Taking the positive part and then summing establishes (28). To conclude that $D_{\text {sim }} \geq D^{\star}$, we need only observe that $D^{\star}$ is a lower bound on the dual objective (4) evaluated at $\left(\tilde{Y}_{m}, \tilde{z}_{m}\right)$. 